\title{
CLINICAL EVALUATION OF CONVENTIONAL GLASS IONOMER VERSUS NANO FILLED GLASS IONOMER RESTORATION OF PRIMARY MOLARS
}

\author{
Abd-Elaziz A.*, El Bayoumy S. ${ }^{* *}$ and Barakat I. ${ }^{* * *}$
}

\begin{abstract}
Aim: The purpose of this study was to clinically evaluate conventional glass ionomer versus nano filled glass ionomer as a restorative material in proximal cavities of primary teeth. Methods: This study included 60 primary molars in 60 children 4-9 years of age who underwent an identical conventional class II cavity preparation the study sample was divided equally into two groups according to the type of filling material group A filled with nano filled glass ionomer, group B filled with conventional glass ionomer The patients were recalled after an interval of 3 months and 6 months for evaluation of restorations using USPHS Cvar/ Ryge Criteria. Results: Nanoionomer was significantly better than conventional glass ionomer with respect to color match at 3 months, 6 months $(P<0.001)$. Nanoionomers were also significantly better than conventional glass ionomer in case of cavosurface marginal discoloration and marginal adaptation $(P<0.001)$ at 6 months. There was no significant difference between the two materials with respect to secondary caries at 6 months $(P>0.05)$. There was no statistical significant difference with respect to anatomical form and postoperative sensitivity $(P>0.05)$. Conclusions: Nanoparticulated resin-modified GIC, which is the latest development in a long history of GIC with improved properties, can be a reliable alternative to the other glass ionomer restorations.
\end{abstract}

\section{INTRODUCTION}

Maintaining deciduous teeth in function until their natural exfoliation is absolutely necessary ${ }^{(1)}$. Restoration in the permanent dentition is different from primary dentition due to the limited lifespan of the teeth and the lower biting forces of children (2). In thinking of restorative objectives for children, one must consider several general categorical objectives; Sealing the cavity, preventing further tooth destruction, rendering the tooth and the tooth-restoration interface caries resistant, and ease of use in a clinical scenario must be included. In addition, the material selected for the procedure must endure the grueling environment of the mouth for the period in which it is intended to be effective ${ }^{(3)}$. The major concern of modern dentistry, mainly for the last decade, has become focused on reducing patients risk for caries, stimulating preventive measures, and preserving tooth structure, indicating, as often as possible, non -invasive conservative techniques instead of proceeding with an invasive healing treatment ${ }^{(4,5,5)}$. The ability of glass-ionomer to release fluoride has been known for a long time and has been a significant factor in their increasing use in dentistry. Fluoride ions released by glass-ionomer cements helped in reduction of demineralization of adjacent enamel, enhancement of its remineralization and prevention of secondary caries by inhibition of microbial growth and metabolism. Quantity of fluoride ions released from the glass-ionomer cements has major importance in definition of their biological activity ${ }^{(7)}$. Nanoionomer is the latest development in a long history of glass ionomer tech-

\footnotetext{
* Dentist at ministry of health

** Professor and Head, of Pedodontics and Oral Health Department Faculty of Dental Medicine Al-Azhar University (Boys - Cairo)

*** Lecturer of Pedodontics and Oral Health Department Faculty of Dental Medicine Al-Azhar University (Boys Cairo)
} 
nology developed by 3M ESPE. Nanoparticulated ionomer is the first resin-modified glass ionomer cement with nanotechnology, combining the benefits of resin-modified light-cure glass ionomer cement (RMGIC) and bonded nanofiller particles. Nanotechnology provides some value-added features not typically associated with glass ionomer restorative materials such as improved polish and aesthetics, abrasion resistance, strength, optical properties, and increased fluoride release. However, there are no documental clinical studies ${ }^{(8,9)}$. Glass ionomers are also accepted as the best dentin replacement materials available for the clinician. However, disadvantages related to glass ionomers; such as lack of strength, prolonged setting time, moisture sensitivity, dehydration, and poor esthetics are reported. Due to these disadvantages of the conventional glass ionomer cement, hybrid versions of the material were introduced. With the application of nanotechnology in this field, Ketac N100 is a new paste/paste nanoinomer with a filler composition of $69 \%$. The glass component of Ketac N100 consists of nanofillers $(5-25 \mathrm{~nm})$ and nanofiller clusters $(1-1.6 \mu \mathrm{m})$. Light activation is required for polymerization. It is claimed to have practical use both in primary and permanent dentition ${ }^{(10)}$.

\section{MATERIALS AND METHODS}

This study was carried out on sixty primary molars selected from sixty children from the Pedodontics Outpatients Clinic, Faculty of Dental Medicine, Al-Azhar University. The age of children was ranged from 4 to 9 years.

\section{Clinical inclusion criteria:}

1. Proximal caries of a primary molar.

2. Vital non exposed pulp of primary molar.

\section{Exclusion criteria}

- Exposed pulp of primary molar.

- Periodontal pathology.
The treatment plan was explained to the parents and their written consent was signed before the study.

\section{Clinical procedures}

Sixty primary molars were randomly assigned into two groups $(n=30)$, according to filling material

Group A-Experimental group Ketak nano Light curing glass ionomer restorative 3M ESPE.

\section{Group B-Control Group: Medicem PROMEDICA}

In each treatment group, class II cavity was achieved with small and medium slow-speed round burs under local analgesia.

In ketac nano group first Use Ketac ${ }^{\mathrm{TM}}$ Nano Light-Curing Glass Ionomer Restorative Primer as a wetting agent on placement instruments which will aid in minimizing handling concerns. Then light cure for 20 seconds then Open nozzle on capsule to $180^{\circ}$ until it's in a straight line with capsule body. Dispense slowly and bleed capsule before dispensing into preparation. Subsequent placement of material following initial placements from capsule must be done within 1.5 minutes. Then apply the material in 2-mm increments to the cavity preparation then light cure for 20 seconds.

In conventional glass ionomer group first on glass slap dispense liquid and powder 1:1 then mix with spatula for one minute maximum, then place the material on the preparation with working time maximum 1.5 minutes.

\section{Follow-up}

Primary molars selected from sixty children were followed up clinically at an interval of 3 months and 6 months for evaluation of restorations using USPHS Cvar/Ryge Criteria for color match, cavosurface marginal discoloration, anatomic form, marginal adaptation, recurrent/secondary caries, and postoperative sensitivity. 


\section{Statistical analysis}

The data collected was tabulated and statistically analyzed. The statistical test used for the study was the Chi-squared $\left(\chi^{2}\right)$ test. The statistical analysis was done in Microsoft Excel software. The Chisquare test was used to test asso1ciation using the following formula:

$\chi^{2}=\Sigma(\mathrm{O}-\mathrm{E}) 2 / \mathrm{E}$

Where $\mathrm{O}=$ Observed value and $\mathrm{E}=$ Expected value

Expected cell count $=\frac{\text { Row total } \times \text { Column total }}{\mathrm{n}}$

$P$ value of 0.05 or less was considered for statistical significance.

\section{RESULTS}

\section{Comparison between group A (ketac nano) and group B (Medifil)}

\section{According to color match:}

Nanoionomer was significantly better than conventional glass ionomerwith respect to color match at 3 months and 6 months (table 1).

\section{According to marginal discoloration:}

There was no significant difference between nano ionomer and conventional glass ionomer with respect to marginal discoloration at 3 months but nanoionomer was significantly better than conventional glass ionomer at 6 months (table 2).

TABLE (1) Comparison of color match scores between the two materials

\begin{tabular}{|c|c|c|c|c|c|c|c|}
\hline \multirow{2}{*}{$\begin{array}{c}\text { Time } \\
\text { interval }\end{array}$} & \multirow{2}{*}{ Grade } & \multicolumn{2}{|c|}{ Ketac nano } & \multicolumn{2}{|c|}{ Medifil } & \multirow{2}{*}{$\mathbf{c}^{2}$} & \multirow{2}{*}{$\mathbf{p}$} \\
\cline { 3 - 6 } & & No. & $\%$ & No. & $\%$ & & \\
\hline \multirow{3}{*}{3 months } & Alpha & 30 & 100.0 & 10 & 33.3 & \multirow{2}{*}{$30.000^{*}$} & $<0.001^{*}$ \\
\cline { 2 - 7 } & Bravo & 0 & 0.0 & 20 & 66.7 & & \\
\hline \multirow{2}{*}{6 months } & Alpha & 27 & 90.0 & 12 & 40.0 & \multirow{2}{*}{$16.484^{*}$} & $<0.001^{*}$ \\
\cline { 2 - 7 } & Bravo & 3 & 10.0 & 18 & 60.0 & & \\
\hline
\end{tabular}

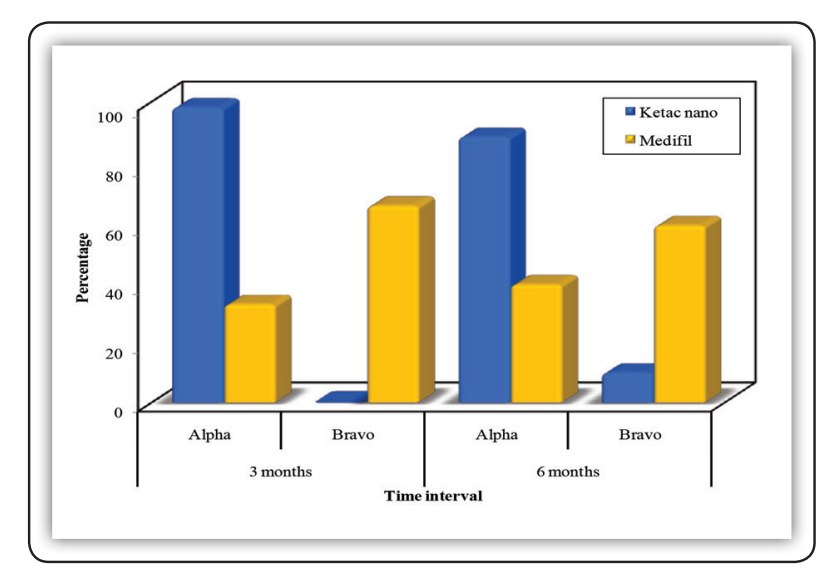

FIG (1) Comparison of color match scores between the two materials

TABLE (2): Comparison of marginal discoloration scores between the two materials

\begin{tabular}{|c|c|c|c|c|c|c|c|}
\hline \multirow{2}{*}{$\begin{array}{c}\text { Time } \\
\text { interval }\end{array}$} & \multirow{2}{*}{ Grade } & \multicolumn{2}{|l|}{ Ketac nano } & \multicolumn{2}{|c|}{ Medifil } & \multirow{2}{*}{$\mathbf{c}^{2}$} & \multirow{2}{*}{$\mathbf{p}$} \\
\cline { 3 - 6 } & & No. & $\%$ & No. & $\%$ & & \\
\hline \multirow{2}{*}{3 months } & Alpha & 30 & 100.0 & 30 & 100.0 & & \\
\cline { 2 - 6 } & Bravo & 0 & 0.0 & 0 & 0.0 & & - \\
\hline \multirow{2}{*}{6 months } & Alpha & 28 & 93.3 & 16 & 53.3 & \multirow{2}{*}{$12.273^{*}$} & $<0.001^{*}$ \\
\cline { 2 - 6 } & Bravo & 2 & 6.7 & 14 & 46.7 & & \\
\hline
\end{tabular}

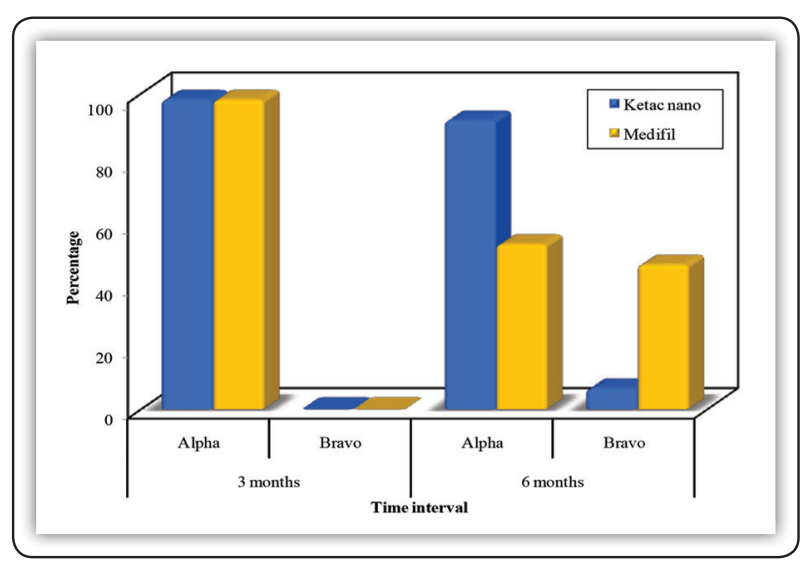

FIG (2) Comparison of marginal discoloration scores between the two materials 


\section{According to marginal adaptation:}

There was no significant difference between nano ionomer and conventional glass ionomer with respect to marginal adaptation at 3 months but nanoionomer was significantly better than conventional glass ionomer at 6 months.

TABLE (3) Comparison of marginal adaptation scores between the two materials

\begin{tabular}{|c|c|c|c|c|c|c|c|}
\hline \multirow{2}{*}{$\begin{array}{c}\text { Time } \\
\text { interval }\end{array}$} & \multirow{2}{*}{ Grade } & \multicolumn{2}{|l|}{ Ketac nano } & \multicolumn{2}{|c|}{ Medifil } & \multirow{2}{*}{$\mathbf{c}^{2}$} & \multirow{2}{*}{$\mathbf{p}$} \\
\cline { 2 - 6 } & & No. & $\%$ & No. & $\%$ & & \\
\hline \multirow{2}{*}{3 months } & Alpha & 30 & 100.0 & 28 & 93.3 & \multirow{2}{*}{2.069} & \multirow{2}{*}{ FE $^{2}=0.492$} \\
\cline { 2 - 6 } & Bravo & 0 & 0.0 & 2 & 6.7 & & \\
\hline \multirow{2}{*}{6 months } & Alpha & 24 & 80.0 & 9 & 30.0 & \multirow{2}{*}{$15.152^{*}$} & $<0.001^{*}$ \\
\cline { 2 - 6 } & Bravo & 6 & 20.0 & 21 & 70.0 & & \\
\hline
\end{tabular}

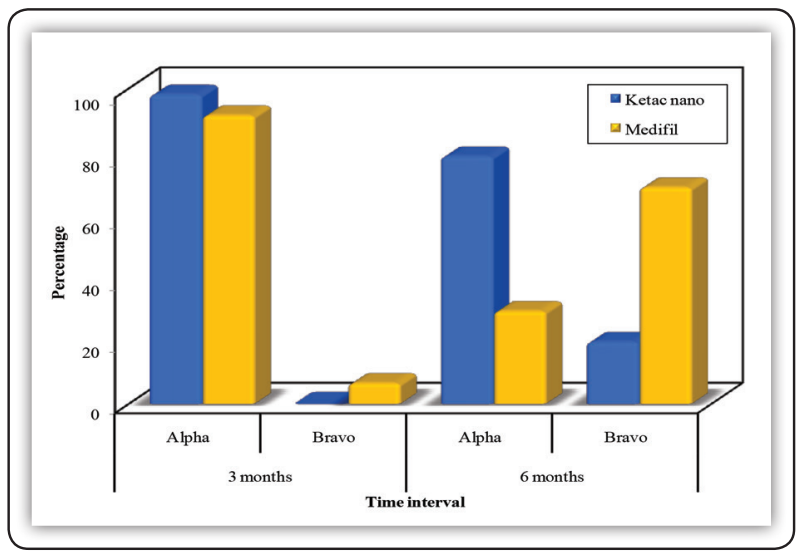

FIG (3) Comparison of marginal adaptation scores between the two materials

\section{DISCUSSION}

One of the important aims in restorative dentistry is to conserve tooth structure during the cavity preparation and removal of caries. Restoration of primary teeth continues to be an important facet of restorative dentistry. The search for an ideal restorative material is a challenge for which dentistry has yet to find a solution. Thus, for each individual clinical situation, dentists must consider certain properties in order to identify the most suitable material. Such properties include biocompatibility, adhesion to the dental structure, absence of marginal leakage, wear and pressure resistance, fluoride release, setting time, facilities related to its manipulation and cost.

In the present study, conventional Glass Ionomer versus nano filled Glass Ionomer restoration of primary molars were clinically evaluated. United States public health service cavar/ryges criteria (11) were used for the evaluation of both materials. Evaluation is done using visual inspection and explorer. In the present study, it was found that Nanoionomer was significantly better than conventional glass ionomer with respect to color match at 3 months and 6 months. In agreement with this result the end result of another research ${ }^{(12)}$ stated that recently, nanotechnologies have been applied to the resin modified glass ionomers in the form of nanoparticles (nanomers) and nanoclusters in fluoroaluminosilicate (FAS) glass. These nanoionomers have been available for clinical use since 2007. The addition of nanoparticles resulted in the aesthetic improvement of the final restoration and polishablilty. On the other hand, another researcher ${ }^{(13)}$ stated that Ketac Nano resulted in a poor color match starting at the baseline evaluation and remained stable thereafter. Although Ketac Nano's surface texture was comparable to that of the nanofilled composite resin, all operators experienced problems with color matching when using the nanofilled RMGIC. In contrast to other RMGICs that darken with time, Ketac Nano restorations were perceived as lighter than the shade selected by the operator prior to starting the restorative procedure. However, the evolution of color mismatch between the restoration and the tooth structure was within the normal range of tooth color and the color stability of the material was acceptable after one year of clinical service in some studies. Nevertheless, the color mismatch between the surrounding tooth structure and the restoration is a rather subjective observation 
because of lighting conditions, and surface staining. (14) Also in the present study, there is no significant difference between nanoionomer and conventional glass ionomer with respect to cavosurface marginal discoloration and marginal adaptation after 3 months, but at an interval of 6 months nanoionomer was significantly better than conventional glass ionomer. The end result of an investigator's (15) study stated that, Microleakage scores of the nanofilled resin-modified glass ionomer (nanoionomer) were not found superior to high viscosity glass ionomer cement (HVGIC) either. Comparison of the dye penetration scores of the two glass ionomer materials tested revealed significant difference in the leakage at the occlusal margin and the marginal sealing of high viscosity glass ionomer was better, while nano-ionomer demonstrated less or similar microleakage than the high viscosity glassionomer cement at the gingival margin. However another investigator ${ }^{(14)}$ stated that, in vitro studies cannot answer questions about in vivo longevity of this tooth colored restorations. Long term results with some of these newly developed materials are lacking and remain controversial as studies report inconsistent clinical results and The criteria used for evaluation in the present study is USPHS criteria (Ryge criteria), and which is the only available criteria widely used for long-term evaluation of restorations, and is considered valid for comparison purpose among studies at different observation periods.

\section{REFERENCES}

1. Parisay I, Ghoddusi J, Forghani M. A Review on Vital Pulp Therapy in Primary Teeth. Iran. Endo. J. 2015; 10:6-15

2. Cho S, Cheng A. A Review of Glass Ionomer Restorations in the Primary Dentition. J. Can. Dent. Assoc. 1999; 65:491-5

3. Berg J. Glass ionomer cements Ped. Dent. - 2002; 24:25
4. El-Motayam K, Fouad W, Youssef R. Assessment and Comparison of Nanoleakage and Resin Tag Length of Three Different Pit and Fissure Sealants: An In-vitro Scanning Electron Microscope Study. J. Americ. Sci. 2013; 9 329-39.

5. Newbrun E. Preventing dental decays. Current and prospective strategies Jam. Dent. Assoc. 1992; 123:68-73.

6. Borsatto C, Corona A, Dibb G, Ramos P, Pécora D. Microleakage of a resin sealant after acid-etching, Er:YAG laser irradiation and air abrasion of pits and fissures. J Clin Med Laser Surg 2001; $19: 83-87$.

7. Selimović-Dragaš M. , Hasić-Branković L, Korać F. In vitro fluoride release from a different kind of conventional and resin modified glass-ionomer cements, Bosn J Basic Med Sci 2013; 13: 197-202

8. Paschoal A, Magalhaes C, Rios D, Abdo C. Oral Health and Preventive Dentistry. Fluoride release of a Nanoparticulated Resin Modified Glass Ionomer Cement.2008; 86: $61-65$.

9. Konde S, Raj S., Jaiswal D. Clinical evaluation of a new material:Nanoparticulated resin-modified glass ionomer cement . J Int Soc Prev Community Dent. 2012; 2: 42-47.

10. Eronat N, Yilmaz E, Kara N. Comparative evaluation of microleakage of nano-filled resin-modified glass ionomer an invitro study. Eur J Dent 2014; 8:50-5.

11. Bayne C. Schmalz G. Reprinting the classic article on USPHS evaluation methods for measuring the clinical research performance of restorative materials Clin Oral Invest .2005;9: 209-214.

12. Zohaib K, Muhammad Z., Saad Q., Advances in Nanotechnology for Restorative Dentistry Materials 2015, 8, 717-731.

13. J Perdiga o, M.Corre^a S. Ciaramicoli, Clinical Research Randomized Clinical Trial of Two Resin-Modified Glass Ionomer Materials: 1-year Results Operative Dentistry, 2012, 37-6, 591-601.

14. Nassar M, Abdulla A, One year clinical follow up of nano filled glass ionomer and composite resin restoration Tanta Dent. J. April 2014; (1): 21-35.

15. Nesrin E, Emir Y, Nazan K, Asli T. Comparative evaluation of microleakage of nano-filled resin-modified glass ionomer: An in vitro study. European Journal of Dentistry, 2014; 8 (4):16-20. 
